\title{
Differences of Angiogenesis Factors in Tumor and Diabetes Mellitus
}

\author{
Shidong Tan' \\ Guangyao Zang' \\ Ying Wang' \\ Zhen Sun' \\ Yalan $\mathrm{Li}^{\mathrm{I}}$ \\ Cheng Lu ${ }^{2}$ \\ Zhongqun Wang (D) \\ 'Department of Cardiology, Affiliated \\ Hospital of Jiangsu University, Zhenjiang, \\ People's Republic of China; ${ }^{2}$ General \\ Office, Affiliated Hospital of Jiangsu \\ University, Zhenjiang, People's Republic \\ of China
}

\begin{abstract}
Angiogenesis, as a process occurring under the regulation of a variety of factors, is one of the important ways of vascular development. It coexists in a variety of pathological and physiological processes. Now a large number of studies have proved that tumor growth, metastasis, and various vascular complications of diabetes are closely related to angiogenesis, and an increasing number of studies have shown that there are many common factors between the two. But angiogenesis is the opposite of the two: it is enhanced in tumors and suppressed in diabetes. Therefore, this review discusses the causes of the phenomenon from the expression of various factors affecting angiogenesis in these two diseases and their effects on angiogenesis in the relevant microenvironment, as well as the application status of these factors or cells as therapeutic targets in the treatment of these two diseases.
\end{abstract}

Keywords: diabetes mellitus, tumor, angiogenesis, differences, tumor microenvironment, hypoxia

\section{Introduction}

Two major diseases threatening human health are diabetes and tumor, among which tumor is a multi-factor and heterogeneous disease, and the incidence of tumor is increasing year by year, bringing more and more medical burden to human beings. Diabetes mellitus is a chronic multi- factor metabolic disease. ${ }^{1}$ According to the statistics, in 2019, about 463 million adults, aging from 20 to 79, suffer from diabetes worldwide, and the vast majority of them are type 2 diabetes patients. It is widely recognized that the metastasis and development of tumors, as well as the clinical symptoms related to diabetes, such as diabetic foot, and retinopathy, all involve a common problem: angiogenesis.

Angiogenesis, considered as a process of new blood vessel formation from preexisting vessels caused by changes in the growth state of endothelial cells and an imbalance between pro- and anti-angiogenic factors, with the pro-angiogenic factors as the predominance ${ }^{2}$ is one of the ways of the development of blood vessels and the other is vasculogenesis. Angiogenesis can be divided into physiological and pathological angiogenesis. Physiological angiogenesis is mainly seen in wound repair and endometrial hyperplasia during the menstrual cycle, ${ }^{3,4,6}$ while pathological angiogenesis is mainly seen in tumors, chronic hepatitis, diabetes, etc. Besides, angiogenesis is mainly divided into two forms intussusception and budding, among which budding is more common, and the angiogenesis mentioned below mainly refers to budding angiogenesis.

Tumor angiogenesis has an unavoidable topic: tumor microenvironment (TME). The tumor microenvironment is composed of extra-cellular matrix (ECM), blood 
vessels, and non-transformed cells such as fibroblasts, adipocytes, glandular cells, and immune cells. ${ }^{1}$ Among them, the hypoxic tumor microenvironment is the key feature of many solid tumor tissues, and tumor cells and tumor microenvironment form a mutually beneficial and coexisting relationship, promoting angiogenesis and facilitating its growth. Although there is blood vessels coselect to promote tumor growth and spread, ${ }^{5,7,8}$ current mainstream research results still indicate that angiogenesis is a necessary condition for rapid tumor growth and metastasis. Moreover, tumor angiogenesis does not occur at the beginning, but after the tumor grows to a certain extent. However, compared with normal new blood vessels, the new blood vessels in tumor tissue are tortuous, disorganized, brittle, and highly permeable. ${ }^{5,10}$

As we all know, the cause of disability and death of diabetic patients is often not diabetes itself, but a variety of vascular complications of diabetes, among which two complications are more special than any others. One of them is retinopathy, initially resulted from degradation and capillary vascular network formation, which then leads to pathological new blood vessels as a compensatory response. ${ }^{11}$ Currently, several relevant literatures have proved that vascular endothelial growth factor (VEGF) plays the most critical role, ${ }^{12}$ and VEGF level will increase with the duration of diabetes. ${ }^{13}$ However, the study of Dan-Yang Chen et al indicated that semaphorin $3 \mathrm{G}(\mathrm{Sema} 3 \mathrm{G})$ can promote the normalization of retinopathy vessels, so Sema3G is likely to become a new target for retinopathy treatment in the future. ${ }^{11}$ The other is atherosclerosis. When complicated by the disease, the proliferation and migration of the activated vascular smooth muscle cells (VSMC), leads to vascular luminal stenosis and local oxygen, ${ }^{14}$ and lack of oxygen, which further results in many pathophysiological processes, such as accumulation of reactive oxygen species (ROS), and hypoxia inducible factor-1 (HIF - 1) and endothelial damage as its result, ${ }^{15}$ VEGF, ${ }^{16}$ long noncoding RNA- H19 (lncRNA - H19) ${ }^{17}$ and so on promote angiogenesis factor increases increasing and leads to pathological blood vessel formation, The unstable easily broken blood vessels and often means the speeding up of the development of atherosclerosis. In addition to the above two complications, diabetes mellitus is characterized by decreased overall expression of angiogenic factors such as stromal cell-derived factor-1 (SDF-1), VEGF, HIF-1, granulocyte colony- stimulating factor (G-CSF), and the receptor CXC chemokine receptor type 4 (CXCR4) in the heart, vascular system and wound, etc. Therefore, on the whole, diabetic patients are still mainly manifested by insufficient angiogenesis. $^{18}$

Tumor and diabetes mellitus themselves have many common metabolic risk factors, such as high insulin, high insulin-like growth factor, etc., and it has been proved that the occurrence and development mechanism of the two are closely related. ${ }^{19,20}$ Therefore, in theory, the two should show similar performances. Then, why the diabetes patients mainly show inhibition of angiogenesis, while the tumor patients show obvious enhancement of angiogenesis, which promotes its development and metastasis, should be analyzed from various factors affecting angiogenesis. Then, after finding these influencing factors, can we artificially create or enhance the effects of these factors that are conducive to angiogenesis in these tumors in the diabetic microenvironment, so as to promote angiogenesis in diabetes? This could be used to treat diabetic foot, a complication caused by inadequate angiogenesis. Correspondingly, of course, it can also be used to treat tumors by inhibiting relevant factors. Or when a diabetic patient suffers from tumor, whether these factors can be adjusted to enhance the effect of chemotherapy drugs, inhibit the growth of tumor, or even achieve the goal of "self-healing".

\section{VEGF}

It is well known that VEGF is the most effective factor in promoting angiogenesis. There are seven isostructural forms of VEGF, among which VEGF-A is a powerful stimulating factor of angiogenesis, and its expression and action are closely related to hypoxia. ${ }^{5,21}$ From the results available now, VEGF is mainly increased in tumors, while mainly decreased in diabetes. The cause of this phenomenon mainly include the following: (1)Hypoxia.: We know from the past that hypoxia is a hallmark of the tumor microenvironment. In diabetes, hypoxia, compared to the tumor, is generally not significant compared to the tumor, unless atherosclerosis is involved. In addition, the longterm hyperglycemia status can cause the decrease of the reactivity of tissue cells to hypoxia. Hypoxia promotes the activation of HIF, thereby upregulating VEGF. Hypoxia also promotes the expression of phosducin-like 3 (PDCL3), which regulates angiogenesis by increasing VEGFR-2 expression. Therefore, it is expected to control tumor angiogenesis by increasing the content of oxygen in the tumor microenvironment, so as to achieve the purpose of inhibiting tumor growth. (2) Hyperglycemia: Hyperglycemia first induces the activation of NADPH 
oxidase to produce ROS, and induces the activity of arginase, which leads to the loss of eNOS activity and then the reduction of NO. Plus, the process produces reactive nitrogen and the toxic effects of hyperglycemia itself. Together, these four factors lead to the destruction of endothelial cells and the decomposition of VEGFR2, leading to reduced production and low utilization of VEGF. In addition, it should not be ignored that chronic high blood glucose can produce AGEs, which can induce VEGF resistance and uncoupling of VEGF and NO caused by high blood glucose itself, resulting in low bioavailability of NO. Therefore, hyperglycemia is an important reason for the low level of VEGF and low angiogenesis in diabetic patients. ${ }^{1,12,21,23,37}$ (3) inflammation: inflammation is characteristic of both common ${ }^{22,23}$ but chronic inflammation of diabetes and the accumulation of the AGEs, inhibits the early inflammatory gene expression, reduces the number of activated macrophages, and damages its function, ${ }^{23}$ resulting in a reduction in the secretion of VEGF levels. ${ }^{12}$ In addition, lack of new blood vessels can lead to other local nutrition supply, and block the migration of inflammatory cells, which makes the ability of local immune cells to produce VEGF more restricted. In the tumor microenvironment, macrophages are transformed into tumor-associated macrophages (TAMS) to secrete VEGF, and the inflammatory mediator cyclooxygenase-2 (COX-2)/ microsomal prostaglandin E synthase1 (mPGES-1) tumor cells are overexpressed to promote VEGF expression. ${ }^{22}$ (4) other pathways: JAK2/STAT3 pathway in the tumor microenvironment, angiotensin II

(ANG-II), metallothionein 2 (MT2) have been proved that they can be induced by VEGF expression; ${ }^{34}$ (5) Different cell types in their microenvironment: retinal pigment epithelial cells, tumor cells, and tumor stromal cells have all been proved to produce a large amount of VEGF. Unfortunately, these VEGF-producing cells are not present in other tissues of diabetes, resulting in the appearance of low VEGF levels.

What is interesting is that current experimental and clinical treatment results show that the efficacy of VEGF treatment in the two diseases is also quite different. In terms of tumor therapy, Bevacizumab hinders the formation of new blood vessels by inhibiting the effect of VEGF-A. ${ }^{24-27}$ However, it is only effective for some cancers. More data show that Bevacizumab has an unsatisfactory therapeutic effect on a variety of tumors, including breast cancer, and even causes rapid progression of many tumors. ${ }^{5,28,29}$ In particular, Andreas Stadlbauer et al used a novel multiparametric MRI and TME mapping to point out: After Bevacizumab treatment, the neovascularized tumor volume increased again from $35.7 \%$ to $39.9 \%$ at the second follow-up. The active tumor volume increased again from $30.2 \%$ to $33.1 \%$ at the 3 rd month after treatment. This may be due to the induction of other vascular growth factors to replace VEGF when anti-VEGF therapy is administered at high doses or for long periods, ${ }^{28}$ or to the fact that the factors leading to metastasis and angiogenesis have not yet been identified. In the treatment of diabetes, VEGF replacement therapy has shown initial results in the treatment of wound healing and reversal of myocardial apoptosis. ${ }^{12}$ Also, for the treatment of retinopathy, the use of anti-VEGF agents in the treatment of this disease is beneficial, ${ }^{4}$ but side effects of systemic administration or topical application of the eye should be avoided.

\section{HIF-I}

HIF-1 is a dimer complex composed of $\alpha$ subunit and $\beta$ subunit, and the synthesis and stable aggregation of HIF$1 \alpha$ is a key link in determining HIF-1 activity. Under hypoxia, HIF-1 $\alpha$ is up- regulated, leading to the expression of angiogenic factors, such as VEGF, angiopoietins 2 (Ang-2), SDF-1, platelet-derived growth factor (PDGF), and microRNA210 (miRNA210). ${ }^{18}$ Besides, it can be further regulated through other pathways including phosphoinositide 3-kinase. ${ }^{18}$ Similar to VEGF, HIF-1 is highly expressed around tumors while low expressed in the diabetes-related microenvironment. This phenomenon can be analyzed from the two aspects of HIF-1 generation and degradation. (1) Generation: Insulin-like growth factor 1 (IGF1)/IGF1R has been reported to activate HIF-1 $\alpha$ transduction in breast tumors. ${ }^{9}$ Moreover, local hypoxia also increases HIF-1 expression. However, in diabetes, high glucose concentration leads to increased hyperoxide level, which induces the accumulation of pyruvaldehyde in cells, and reduces the protein level and transcriptional activity of HIF-1 $\alpha .^{18}$ (2) Degradation: Under hypoxic conditions, HIF- $1 \alpha$ is not degraded but binds to the HIF- $1 \beta$ subunit to form HIF-1. On the other hand, interleukin -6 (IL-6) secreted by tumor cells can inhibit the expression of p53, thus inhibiting HIF-1 $\alpha$ degradation. ${ }^{31}$ In diabetes, high glucose promotes HIF-1 $\alpha$ degradation through multiple pathways, resulting in decreased HIF- $1 \alpha$ expression.

The reasons why HIF- $1 \alpha$ is decreased in diabetic patients with both hypoxia and hyperglycemia at the wound, are based on the following facts: the environment 
in the early stage of wound formation will stimulate the production of HIF-1 $\alpha$, but hyperglycemia reduces the release of SDF- 1 and VEGF. It can be speculated that there are two reasons for this phenomenon: (1) Other key downstream links of angiogenesis are damaged, leading to HIF-1 $\alpha$ accumulation in the wound area, and HIF-1 $\alpha$ transcription and expression are inhibited by negative feedback.(2) Besides, the toxicity of high glucose itself on HIF-1 $\alpha$ further reduces the expression of HIF-1 $\alpha$, which leads to the decrease of HIF-1 $\alpha$ in the local late wound of diabetic patients. On the other hand, in addition to promoting angiogenesis, HIF-1 $\alpha$ helps acidify the microenvironment of tumor cells, inhibits citrate cycle (TCA cycle) metabolism, and enhances genes in the glycolysis pathway, thereby supporting tumor growth and metastasis. $^{32}$ In terms of treatment, Lan Lin et al have demonstrated that

BML-111 can inhibit HIF-1 and COX-2 by utilizing VEGF or $\mathrm{CoCl} 2$ to mimic tumor microenvironment in vitro. In contrast, HIF-1 is more difficult to treat diabetes because high expression of HIF-1 $\alpha$ may induce or aggravate retinopathy. However, HIF-1 $\alpha$ has also been reported to be used in patients with no-option critical limb ischemia. ${ }^{18}$

\section{NO}

NO can be synthesized by three nitric oxide synthases (NOS), and NO is mainly produced by endothelial nitric oxide synthase (eNOS). ${ }^{33}$ Under physiological conditions, NOS in vascular endothelial cells can synthesize small doses of NO, while HIF-1, VEGF, and SDF-1 can further increase NO release through various pathways under ischemia and hypoxia conditions. The effect of NO may depend on the environment and cell type and concentration. ${ }^{33}$ Low concentrations of NO increases intracellular cyclic guanosine monophosphate (cGMP) in response to VEGF stimulation of angiogenesis, while high concentrations inhibits angiogenesis. $^{34}$ What is interesting is that not only VEGF and HIF-1 regulate the synthesis and secretion of NO in various ways, ${ }^{34,35}$ but also eNOS and NO in response are believed to increase VEGF expression and HIF-1 activity, thereby stimulating angiogenesis. ${ }^{34,36}$

Similar to the above two factors, NO is also highly expressed in tumors and low-expressed in diabetes. The reasons can be attributed to different characteristics of the microenvironment and different substances in the microenvironment. (1) The characteristics of the microenvironment are different: in the hypoxic tumor microenvironment, high expression of HIF can promote high expression of NOS, synthesis, and release of NO. Under a high glucose environment, various pathways lead to the down-regulation of eNOS expression and the reduction of phosphorylation, the reduction of VEGF release, the uncoupling of VEGF and $\mathrm{NO}$, and the induction of arginase consumption of $\mathrm{NO}$ precursor arginine, leading to the reduction of NO synthesis and release and low bioavailability ${ }^{12,37}$ (2) Different substances in the environment: VEGF, fibroblast growth factor (FGF), and other factors are released around tumors, which can activate relevant signaling pathways and lead to the activation of eNOS. ${ }^{6}$ High insulin in diabetic patients inhibits the endothelial production of bioavailable NO. Papers have shown that both the reduction of endothelial precursor cells (EPCs) quantity and the inhibition of migration caused by hyperglycemia are closely related to the decrease of NO production or abnormal activity. However, NO has multiple effects on tumor biology, depending on the concentration of NO in the tumor tissue, duration of exposure, NOS activity level, tumor type, nature of the surrounding microenvironment, and sensitivity of related cells to NO. ${ }^{33}$ So different experiments have yielded different results: Tijana Suboticki et al proved through DNA Sequencing, Isolation ofTotal RNA, RT-PCR, Western Blot and other methods that there is a positive correlation between VEGF and eNOS levels in granulocytes of MPNs. However, Amir M. Alsharabasy et al explored the regulatory effect of NO on MMPs. ${ }^{33,34,38}$ In the treatment of diabetes, chronic morphine treatment ${ }^{39}$ and Metformin ${ }^{40}$ have achieved good results in animal models. However, in the treatment of cancer, few reports have been on the therapeutic effect achieved by inhibiting NO. This may due to the multiple pathways and factors in the tumor-related environment, leading to an increase in NO expression. Therefore, when all pathways cannot be all blocked, other pathways may be likely to compensate, resulting in an insignificant decrease in NO.

\section{MMPs}

Matrix metalloproteinases (MMPs), a family of $\mathrm{Zn} 2 \mathrm{p}$ dependent endopeptidases, are responsible for the digestion of a variety of extracellular matrix (ECM) components, also have the ability to promote endothelial cell proliferation and migration, and are important pro-angiogenic factors. ${ }^{6,45}$ Moreover, the activity of these MMPs is regulated in various ways, such as NO, tissue inhibitor of metalloproteinases (TIMPs), etc. ${ }^{33,46}$ Also, activated protein1 (AP-1) and NF$\mathrm{kB}$ are believed to be related to the expression of MMP-9. ${ }^{47}$ 
However, MMP-9 may harm angiogenesis by producing angiostatin. $^{47}$

The hypersecretion of MMPs is due to hypoxia, the direct secretion of tumor-associated inflammatory cells, the carrying of some tumor exosomes, and the regulation of $\mathrm{NO}$ in some tumors [2,29,31,38,48,49,50,51,52,]. However, in diabetes mellitus, the conclusions seem contradictory: it is generally believed that hyperglycemia impresses the expression of MMP-1, MMP-2, and MMP- 9 in smooth muscle cells. However, Meijia Zhu et al showed more expression of MMP-9 when ischemia was combined with diabetes by using immunohistochemistry, Western blot and SDS-Page zymography. ${ }^{47}$ It may be that the combination of ischemia and diabetes activates another pathway that increases MMP-9 production. The key mechanism of EPCs reduction in diabetes is also believed to be closely related to MMP-9. ${ }^{41,47}$ In tumors, MMP-1, MMP-2, and MMP-9 are involved in metastasis, in which MMP-9 also induces angiogenesis and tumor growth in some tumors. ${ }^{38,45,48,49}$ In some tumors, Circular RNAs (CircRNAs) are also involved in ECM remodeling by regulating the expression of MMPs. ${ }^{62}$ MMPs related therapy: blocking urokinase- type plasminogen activator (UPA) and MMP-9 in tumors has been shown to inhibit TAM-induced invasion; ${ }^{49}$ In diabetes, Amadori-glycated phosphatidylethanolamine (Amadori-PE) stimulates the secretion of MMP-2 and promotes neovascularization. ${ }^{45}$

\section{FGF}

It is now widely believed that VEGF and FGF are the main inducers of angiogenesis, but FGF and VEGF are different in that VEGF acts more selectively, while the FGF family shows biological effects on various cell types. $^{57}$ Similar to VEGF, FGF also exerts its effects by binding to receptors. ${ }^{58}$ Besides, FGF, transforming growth factor (TGF- $\beta$ ), and PDGF can jointly promote VEGF expression. Basic FGF (bFGF) and prostaglandin E1 (PGE1), members of the FGF family, are believed to have synergistic effects, and the effect depends on the number of up-regulated VEGF. ${ }^{59}$ Besides, endocan is also believed to have a regulatory effect on bFGF. ${ }^{58}$

Due to the secretion of bFGF by tumor cells themselves, TAMs, and cancer associated fibroblasts (CAFs), the carrying of exosomes, and the promoting effect of COX-2/ mPGES-1, FGF is highly expressed in the microenvironment of various tumors. ${ }^{6,31,32,38,50,60}$ However, in diabetes, different experiments have drawn different conclusions: some believe that the expression of FGF is increased, while others believe that there is no abnormality. ${ }^{35}$ This may be related to different measurement methods and specific microenvironment differences in different patients and in different parts of the same patient. In tumors, the anti-angiogenesis of interferon and the development of drug resistance in some tumors $^{25}$ may be related to bFGF, especially the occurrence and development of endometrial cancer. ${ }^{8}$ In the early stage of diabetes, bFGF may increase, but in the long-term highglucose environment, bFGF can covalently bind with reducing sugar to form glycated fibroblast growth factor (gFGF), and this process may be an important reason for the decline of EPCs function. FGF-related therapies in tumors: HRGP attenuates the effects of FGF; in diabetes: PGE1 can effectively improve the angiogenic characteristics of bFGF in patients with diabetes, ${ }^{59}$ and Leptin can increase the release of bFGF, ${ }^{20}$ so these substances may be used as related therapeutic drugs. Besides, preventing the formation of gFGF or reducing gFGF may become a new target for the treatment of diabetic microangiopathy.

\section{PDGF}

There are four monomers in the PDGF family, and every monomer, playing an important role in promoting angiogenesis and angiogenesis maturation, forms homodimer through disulfide bonds. Among those monomers, PDGF$\mathrm{B}$ can induce endothelial cell proliferation, migration, and stability of the vascular structure. PDGF-D can regulate the VEGF signal. ${ }^{31}$ However, excessive PDGF function is also not conducive to angiogenesis, which may be related to increased pericyte recruitment and vascular maturation of PDGF-B, or it may be that a high concentration of PDGF-B has an inhibitory effect on pericytes. Besides, neuropilin 1 (Nrp1) is also believed to have a certain connection with PDGF.

As the long-term stimulation of diabetes itself, the activation of PKC and other factors, promote the synthesis of PDGF is promoted to, increases the level of PDGF in the retina and kidney of diabetic patients, further advance the formation of new blood vessels by stimulating intercellular adhesion molecule - 1 (IGAM-1) and other ways, and aggravate microvascular-related lesions. The tumor microenvironment also contains abundant PDGF, possibly for the following reasons: $\mathrm{TAMs}^{32,49,50,63}$ and $\mathrm{CAFs}^{31,46}$ can secrete PDGF, and on the other hand, active HIF-1 can increase the release of PDGF under hypoxia conditions. ${ }^{35}$ PDGF promotes tumor angiogenesis through its effects and co-action with other factors, ${ }^{8}$ but overexpression of PDGF-B significantly inhibits tumor growth in vivo. This may be related to other signaling pathways that have not 
yet been discovered, such as the negative feedback inhibition of PDGF on VEGF and CAFs. Overexpression of PDGF-B activates one or more of these pathways, resulting in an inhibitory effect. Dual blocking of PDGFR $\beta$ / VEGFR is effective in preclinical and clinical studies of tumors and has also been shown to be effective with imatinib or by blocking PDGF receptors.

\section{ANG}

Ang is also an important pro-angiogenic factor that regulates angiogenesis by binding to Tie- 2 receptors. ${ }^{38,68}$ The effect of Ang-2 is influenced by the microenvironment: when VEGF is highly expressed, angiogenesis is promoted; when VEGF is deficient, competitive antagonism of Ang-1 reduces neovascularization by promoting vascular maturation and stabilizing neovascularization. Serum Ang-2 levels are closely related to the severity of diabetic retinopathy, and the longer the course of diabetes, the higher the level of Ang-2 levels. Synergistic with VEGF, Ang-2 levels promotes angiogenesis and the occurrence of retinopathy. Similarly, high concentrations of VEGF in the tumor microenvironment promote Ang-2 as angiogenesis. ${ }^{5}$ Moreover, Ang-2 is a potential surrogate marker of liver metastasis in patients with metastatic colorectal cancer. ${ }^{30}$ In terms of tumor therapy, inhibition of VEGFR2-Ang-2 by dual inhibition has shown good efficacy in preclinical models. ${ }^{68}$

\section{PAI- I}

Plasminogen activator inhibitor-1 (PAI-1), a major physiological inhibitor of endogenous plasminogen activators, is synthesized by perivascular cells and is extremely unstable in vitro and in vivo, but can be stabilized by binding to vitronectin and affects angiogenesis by interacting with the u-PA receptor. ${ }^{20}$ PAI-1 is highly expressed in both diabetes and tumor, but its effects are different: in diabetes, it impresses angiogenesis and promotes the formation of atherosclerotic plaques; ${ }^{69}$ however, its effect on tumor has a dual nature: PAI-1 promotes angiogenesis by inhibiting PA at the physiological level; However, it can inhibit tumor angiogenesis at the pharmacological level, ${ }^{20}$ but the mechanism of this phenomenon still needs further clarification.

\section{Pericytes}

It is now widely recognized that pericyte recruitment and attachment play a very important role in the stability of neovascularity. ${ }^{5,55,70}$ Pericyte loss in diabetic patients may be related to PDGF-B secretion, ${ }^{8}$ ROS production, and AGEs production, ${ }^{45}$ and it is believed that pericyte loss is also closely related to diabetic retinopathy and diabetic nephropathy. ${ }^{5,62}$ Pericytes are sparse in the tumor microenvironment, which is conducive to tumor invasion and metastasis. ${ }^{68}$ Moreover, tumor-associated cells (TACs) support angiogenesis, a process in associated with pericytes as well. ${ }^{51}$

\section{TNF- $\alpha$}

Tumor necrosis factor-alpha (TNF- $\alpha$ ) can stimulate the release of angiogenesis promoters such as bFGF and VEGF-A and increase the transcription of the VEGFR-2 gene. Meanwhile, NF- $\kappa B$ has also been shown to be downstream of $\mathrm{TNF}-\alpha,{ }^{70}$ so it has the effect of promoting angiogenesis. The circulating level of TNF- $\alpha$ is increased in diabetic patients, ${ }^{20}$ and TNF- $\alpha$ is also highly expressed in tumors, but the reason for the increase in diabetic patients still needs further study. TNF- $\alpha$ may be produced by many different cells during tumor elevation, including M2-type macrophages, TAMs, tumor cells, etc., $32,52,71,72$ and it has been shown that TNF- $\alpha$ promotes tumor angiogenesis. $^{71}$

\section{TGF- $\beta$}

It is well known that TGF- $\beta$ is also one of many angiogenesis regulators. It has a strong influence on angiogenesis through PAI-1 regulation, ${ }^{20}$ VEGF expression, ${ }^{21}$ the promotion of new vascular stability, ${ }^{38}$ and the regulation of both the proliferation and migration of vascular smooth muscle cells. Due to the secretion of exosomes released by some tumors containing TGF- $\beta,{ }^{38} \mathrm{CAFs},{ }^{31} \mathrm{TAMs}^{49,50}$ ), T regulatory cell, and other cells, TGF- $\beta$, in a state of high expression in tumors, promotes tumor angiogenesis and has the role of transforming surrounding fibroblasts into CAFs. ${ }^{8}$ Besides, Endoglin from the TGF- $\beta$ family of receptors. It is also used in 133 patients with Colorectal cancer by ELISA of soluble Endoglin and VEGF, immunohistochemistry, total RNA extraction, cDNA synthesis, and quantitative real-time PCR proved that it was closely related to tumor angiogenesis. ${ }^{73}$ In diabetes, glucose, directly and indirectly, stimulates the expression of VEGF-A in podocytes through TGF- $\beta$, and the increase of VEGF-A inhibits the expression of TGF- $\beta$, which is a negative feedback mechanism. ${ }^{21}$ Moreover, HIF-1 upregulation of VEGF in ischemic diabetes is directly or indirectly regulated by $31 \mathrm{TGF}-\beta{ }^{12}$ 


\section{ROS}

ROS plays an important role in the control of apoptosis and proliferation, in which a moderate increase in ROS level is conducive to cell proliferation, while excessive ROS induces apoptosis, by a mechanism that may be related to intracellular Ca2+ inflow, and CYP450 can increase oxidative stress and induce cytotoxicity together with $\mathrm{ROS},{ }^{53}$ and ROS is involved in the generation of blood vessels. ${ }^{54,55}$

It is different from other factors previously introduced: both diabetes and tumor-related microenvironment are highly expressed, and the mechanism of this phenomenon is related to the following two aspects: (1) Microenvironment characteristics: hypoxia can promote high ROS in the tumor microenvironment; ${ }^{51}$ high glucose induces and activates nicotinamide adenine dinucleotide phosphate (NADPH) oxidases to produce ROS. $^{37}$ (2) Induction of other substances: Tumor-associated TNF- $\alpha$ can increase ROS formation. ${ }^{20}$ Although ROS are highly expressed in both, they seem to have opposite effects. In diabetes, VEGF resistance and podocyte extirpation are closely related to high ROS, and the reduced expression of SDF-1 and VEGF in the heart and vascular system of diabetes is also believed to be related to the excessive production of ROS. ${ }^{41}$ But in tumors, ROS through the way such as activation of NF-kB participate in tumor angiogenesis. ${ }^{54}$ In terms of tumor treatment, decylubiquinone and Benznidazole have anti- tumor effects by inducing ROS overproduction, while Brain angiogenesis inhibitor 1 (BAI1) has been shown to inhibit ROS production and is expected to achieve therapeutic effects. ${ }^{53,54}$ Therefore, in general, both treatments, raising basic ROS levels to toxic levels and reducing ROS levels, are effective targets for tumor therapy. In the treatment of diabetes, Epicatechin has been proven to activate eNOS and has achieved a certain efficacy. ${ }^{56}$

\section{SDF-I/ CXCR4 (a Receptor of SDF-I)}

We have previously mentioned that HIF-1 expression is increased in hypoxia, resulting in increased levels of downstream angiogenic factors, of which SDF-1 is a key factor. SDF-1 can promote VEGF synthesis, inhibit EPCs apoptosis, ${ }^{41}$ and cooperate with endothelial progenitor cells to promote angiogenesis. ${ }^{18} \mathrm{SDF}-1 / \mathrm{CXCR} 4$ signaling can also regulate the mobilization and homing of hematopoietic stem cells and circulating angiogenic cells and plays an important role in the recruitment of bone marrow cells. $^{18,42}$

Similarly, the high level of SDF-1 in tumors and the low level in diabetes are also related to the characteristics of these two diseases in the microenvironment and other pathways.(1) Microenvironmental characteristics: hyperglycemia can reduce the release of SDF-1, and this inhibitory effect increases with the increase of blood glucose. Concerning tumors, hypoxia itself increases CXCR4 expression in the associated cells or modulates CXCR4 expression through HIF-1 and VEGF. ${ }^{42}$ (2) Another pathway: SDF-1 expression is reduced in diabetes due to inhibition of EP3 signal; ${ }^{42}$ in tumors, CAFs have been shown to secrete SDF- $1,{ }^{8}$ and some exosomes of tumor cells are rich in CXCR4 chemokine receptors. ${ }^{38}$ These factors all lead to high levels of SDF-1 high expressing CXCR4 of relevant cells in the tumor microenvironment, while in a low expression state in diabetes. Relevant treatment options for SDF - 1 in the treatment of diabetes has achieved gratifying results: Rosiglitazone, ${ }^{43}$ transplantation of adipose-derived stem cells, ${ }^{42,44}$ and EP3 (a prostaglandin E receptor subtype) excited agent all have achieved satisfactory curative effect on diabetes mice. Besides, insulin treatment can significantly improve the level of plasma VEGF and SDF - 1, so this may mean that increasing insulin levels, and taking good control of blood sugar levels, are likely to increase angiogenesisrelated factor, thus improving related ischemic symptoms.

\section{AGEs}

AGEs, produced by non-enzymatic glycation of reducing sugars with free amino acids on macromolecules such as proteins and nucleic acids, exert corresponding effects through binding to their receptor (RAGE) receptors. ${ }^{23,37}$ Under high glucose conditions, the production of 3deoxyglucosidone ${ }^{37}$ and also the deficiency of Glyoxalase 1 (Glo1) $)^{23}$ preventing the accumulation of AGEs will lead to the increase of AGEs levels.

Elevated AGEs concentrations are known to lead to vascular dysfunction and impaired angiogenesis in diabetes. The mechanism of this phenomenon may be that AGEs can directly activate the p38 and extracellular signal-regulated protein kinases 1 and 2 (ERK1/2) signaling pathways through binding to their receptors and induce VEGF's resistance. AGEs activate the MAPK pathway and promote the apoptosis of EPCs. However, Jeong-Ho Oak et al reported that AGEs can also activate angiogenesis and play a positive 
role in the development of diabetic microangiopathy. ${ }^{45}$ So whether AGEs play a promoting or inhibiting role may depend on the specific environment in which they act. Aminoguanidine has shown promising results in the treatment of multiple diabetic complications by inhibiting AGEs. Besides, AGEs themselves also have the effect of promoting angiogenesis, and the concentration of AGEs is increased in the high glucose environment. In theory, it should be easier to achieve the therapeutic effect by inducing its positive promoting effect on angiogenesis.

\section{Wnt/ $\beta$-Catenin}

Both Wingless and Int-related protein (Wnt) and $\beta$-catenin are multifunctional proteins, in which the expression of $\beta$ catenin is necessary for Sema3G-mediated pathological vascular remodeling mediated, and the $\mathrm{Wnt} / \beta$-catenin signaling pathway formed by both can prevent autophagy and apoptosis, and then promote angiogenesis, ${ }^{64,77}$ etc. However, with the absence of Wnt ligands in this pathway, $\beta$-catenin degrades. ${ }^{65}$

In diabetes, continuous blood glucose elevation can activate the $\mathrm{Wnt} / \beta$-catenin signaling pathway in the heart, retina, kidney, etc., thereby accelerating the pathophysiological processes. Similarly, $\beta$-catenin is overactive in some tumors, is involved in promoting angiogenesis and metastasis. $^{38,65}$ Wnt4 is highly expressed in colorectal cancer and converts NFs to CAFs through activation of the Wnt4/ $\beta$-catenin pathway ${ }^{66}$ Similarly, WNT2 has been shown to promote CAFs migration, ECM modification, and angiogenesis in colorectal cancer. ${ }^{67}$ Recognized as an important target for cancer therapy, the $\mathrm{Wnt} / \beta$-catenin pathway, with Ipafricept bindings Wnt ligand to the fusion protein of Wnt signaling, and has achieved good results when used in combination with standard chemotherapy. ${ }^{65}$

\section{Exosome}

As nanoscale extracellular vesicles first discovered in the supernatant of sheep red blood cells, exosomes can be secreted by various types of living cells and exist in various body fluids. ${ }^{61}$ This vesicle, initially regarded as a "waste product" produced by cells, has been found to contain a variety of proteins, lipids, DNA, and RNA involved in a variety of biological processes. ${ }^{62}$

The role of exosomes in regulating tumor angiogenesis has been reported many times, but different tumors and different types of exosomes have different mechanisms of action: exosomes of chronic myeloid leukemia cells promote angiogenesis through direct interaction with endothelial cells (ECs). ${ }^{38}$ Exosomes secreted by melanoma cells can induce the formation of CAFs and trigger their pro-angiogenic effects. Besides, hypoxia can promote the secretion of exosomes in multiple myeloma and lung cancer. $^{38,52}$ The number of serum exosomes in diabetic mice also significantly increases, and the number of exosomes released by glomerular mesangial cells is positively correlated with glucose concentration. However, different from tumors, exosomes secreted by these cells (such as cardiomyocytes) in diabetes are more inclined to inhibit angiogenesis by reducing NO production in endothelial cells and other mechanisms, thus promoting the occurrence and development of diabetes. Besides, the manifestation of tumor- associated exosomes has also become one important targets of oncology therapy. It has now been proved that manumycin A and Carboxyamidotriazole orotate inhibit the angiogenesis induced by exosomes by inhibiting their generation or action. ${ }^{38}$ Moreover, some exosomes can be used to improve the drug resistance of tumors, and certain substances in exosomes are also considered as auxiliary diagnostic indicators. However, because exosomes contain too many substances and their mechanism of action is complex, it is still difficult to implement them in clinical practice.

\section{EPCs}

EPCs, as primitive cells originating from bone marrow, can differentiate into vascular endothelial cells with the potential to proliferate and migrate. In the process, VEGF may play a key role. ${ }^{12}$ EPCs can secrete VEGF, bFGF, and other cytokines to promote angiogenesis. ${ }^{89}$ Besides, EPCs can stabilize the intima of blood vessels and repair damaged blood vessels. In a diabetic state, due to increased ROS production, the combination of inhibited phosphorylation of eNOS, decreased secretion of VEGF and SDF-1, production of gFGF, and inhibitory effect of AGEs on EPCs, all result in the abnormal function, and quantity of EPCs and end up with diabetic vascular complications. ${ }^{40}$ Therefore, an increase in the number and/or activity of autologous EPCs is considered a promising treatment strategy for angiogenesis-related diseases. However, the effect of increasing the number of diabetic EPCs and improving functional impairment by culture is not ideal. ${ }^{74}$ Therefore, allograft seems to be a better option, and studies have shown that transplanted EPCs can integrate into damaged blood vessels and secrete growth factors to promote angiogenesis. ${ }^{89}$ However, for allotransplantation, immune rejection is also a problem 
that cannot be ignored, so the relevant treatment plan still needs to be further optimized.

\section{TAMs}

TAMs are a large family of macrophages ${ }^{49}$ and are the most abundant population of tumor-infiltrating immune cells in TME. ${ }^{29,49,63} \mathrm{M} 1$ or M2 macrophages are induced by Th1 and Th2 cytokines, respectively, are the two main cell subtypes of TAMs. Moreover, the two effects are opposite: M1 macrophages are mainly involved in killing tumor cells, while M2 macrophages can promote angiogenesis. $^{29,31,49}$ Moreover, TAMs are regarded as a major source of VEGF-A. ${ }^{60}$

Some data have shown that Sema4a, ${ }^{75}$ VEGF, ${ }^{68}$ and HIF$1^{29}$ can promote TAMs recruitment to further advance angiogenesis. Besides, tumor cells, HIF-1, IL-10, lactic acid, and pyruvate in the hypoxic environment are thought to activate the pro-angiogenesis process in TAMs. ${ }^{50}$ Moreover, the number of TAMs in colon cancer is positively correlated with the number of blood vessels. ${ }^{49}$ Treatment regimens for TAMs are mainly divided into four directions: ${ }^{49}$ removal of macrophages, restriction of TAMs recruitment, inhibition of TAMs activation, and increase of M1 and decrease of M2. The last regimen has achieved results in mouse models of breast cancer: After a third dose of trastuzumab was given, the percent of M1 macrophages significantly increased from $13.38 \pm 3.65 \%$ to $31.07 \pm 2.9 \%(\mathrm{P}=0.02)$, and comparison of vessel maturation and percent M1 macrophages from flow analysis revealed a significant positive linear correlation $(\mathrm{R}=$ $0.33, \mathrm{P}=0.04){ }^{29}$

\section{CAFs}

CAFs are an important part of the tumor microenvironment, ${ }^{5,46,51,66,76}$ and one of the most abundant cell types in stromal cells of various tumors, including breast cancer. ${ }^{46,76}$ In essence, CAFs are heterogeneous fibroblasts activated by various stimuli (such as PDGF, TGF- $\beta$, VEGF, IL-6, etc.). 5,31,46,51 Besides, lncRNA, miRNA-9, miRNA-200, and Wnt4 / $\beta$-catenin signaling pathways also play this role, ${ }^{66,76,77}$ while Wnt2 plays a role in promoting CAFs migration. ${ }^{67}$

CAFs can secrete VEGF, FGF, and other factors, 5,31 up-regulate the expression of MMPs and mediate the degradation of ECM (, ${ }^{51}$ and recruit endothelial progenitor cells into tumor stroma to promote tumor angiogenesis. ${ }^{8}$ Besides, CAFs have also been shown to regulate the tumor microenvironment, promote tumor growth, support tumor cell migration, and increase tumor invasion. ${ }^{4,51,66,76}$ Therefore, CAFs may also become a new therapeutic target for tumors.

\section{Conclusions and Perspectives}

Angiogenesis is a complex process regulated by multiple factors, in which VEGF plays a major role. In the tumor-

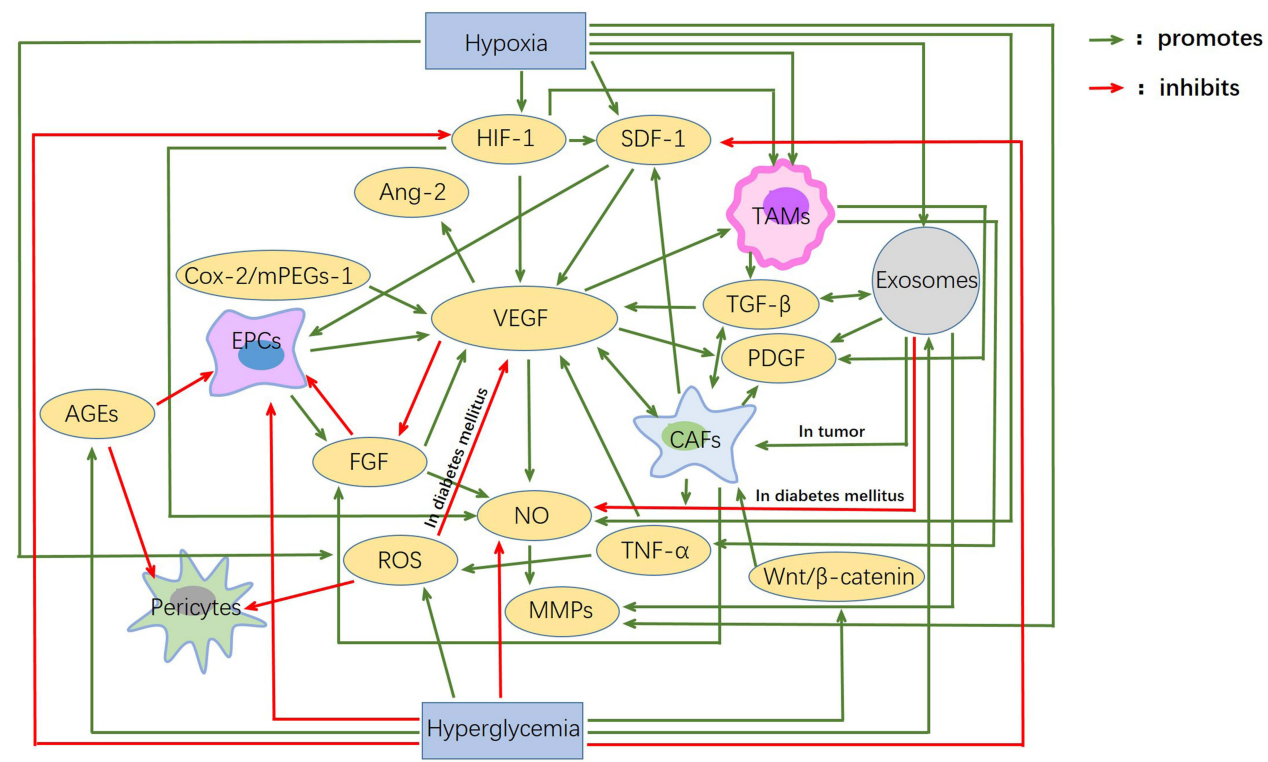

Figure I Interactions between angiogenic factors in diabetes mellitus and cancer. Hypoxia and hyperglycemia are the main characteristics of the microenvironment of tumor and diabetes mellitus. Hypoxia mainly promotes angiogenic factors, while hyperglycemia mainly inhibits them. VEGF, HIF-I, SDF-I, Ang-2, FGF and so on are the main factors affecting angiogenesis. EPCs, Pericytes, TAMS, CAFs, etc. These are the main cells involved in angiogenesis. These factors and cells are closely related to each other. It is the presence of these connections and pathways that account for the huge differences in angiogenesis between diabetes mellitus and tumors. 
associated microenvironment, angiogenesis is significantly enhanced and promoted, while in the diabetes-associated microenvironment, angiogenesis is mainly inhibited to varying degrees, leading to a variety of vascular complications.

Hypoxia is the main inducement of angiogenesis in tumor tissue. Hypoxia can promote transcription activation of HIF, thereby up-regulating the expression of various pro-angiogenesis factors and promoting angiogenesis.
Besides, CAFs, TAMS, regulatory $\mathrm{T}$ cells, and tumorassociated exosomes secrete or carry various proangiogenic factors, such as VEGF, TGF- $\beta$, IL-10, IL-35, and microRNA, etc., and then promote angiogenesis through various pathways and mechanisms, thus promoting the occurrence and development of tumors. The decrease of angiogenesis in diabetes is mainly due to the direct or indirect toxicity of long-term hyperglycemia, which leads to endothelial dysfunction and the decline of

Table I New Advances of Angiogenesis Factors in Tumor and Diabetes

\begin{tabular}{|c|c|c|}
\hline Factors Influencing Angiogenesis & Tumor & Diabetes Mellitus \\
\hline VEGF $^{\prime}$ & high expression, promotes angiogenesis & low expression, promotes angiogenesis \\
\hline HIF-I $I^{2}$ & high expression, promotes angiogenesis & low expression, promotes angiogenesis \\
\hline $\mathrm{NO}^{3}$ & high expression, promotes/inhibits angiogenesis & low expression, promotes angiogenesis \\
\hline SDF- $1^{4} / \mathrm{CXCR} 4^{5}$ & high expression, promotes angiogenesis & low expression, promotes angiogenesis \\
\hline $\mathrm{COX}-2^{6} / \mathrm{mPGES}-1^{7} / \mathrm{PGE}^{8}$ & high expression, promotes angiogenesis & low expression, promotes angiogenesis \\
\hline$M M P^{9}-1$ & high expression, promotes angiogenesis & low expression, promotes angiogenesis \\
\hline MMP-2 & high expression, promotes angiogenesis & low expression, promotes angiogenesis \\
\hline MMP-9 & high expression, promotes /inhibits angiogenesis & high/low expression, promotes /inhibits angiogenesis \\
\hline $\operatorname{ROS}^{10}$ & high expression, promotes angiogenesis & high expression, inhibits angiogenesis \\
\hline FGF $^{\prime \prime}$ & high expression, promotes angiogenesis & high expression/normal, promotes angiogenesis \\
\hline PAI-I ${ }^{12}$ & high expression, promotes /inhibits angiogenesis & high expression, inhibits angiogenesis \\
\hline TNF- $\alpha^{13}$ & high expression, promotes angiogenesis & high expression, promotes angiogenesis \\
\hline $\mathrm{TGF}^{14}-\beta$ & high expression, promotes angiogenesis & high/low expression, promotes angiogenesis \\
\hline Exosomes & production increased, promotes angiogenesis & production increased, promotes /inhibits angiogenesis \\
\hline PDGF $^{15}$ & high expression, promotes /inhibits angiogenesis & high expression, promotes angiogenesis \\
\hline $\mathrm{Wnt}^{16 / \beta} /$-catenin & high expression, promotes angiogenesis & high expression, promotes angiogenesis \\
\hline Ang- $2^{17}$ & high expression, promotes angiogenesis & high expression, promotes angiogenesis \\
\hline$|G F|^{18}$ & high expression, promotes angiogenesis & low expression, promotes angiogenesis \\
\hline Leptin & high expression, promotes angiogenesis & high expression, promotes angiogenesis \\
\hline IGAM-I ${ }^{19}$ & high expression, promotes angiogenesis & high expression, promotes angiogenesis \\
\hline $\mathrm{Nrpl}^{20}$ & high expression, promotes angiogenesis & low expression, promotes angiogenesis \\
\hline Semaphorins & high expression, promotes/inhibits angiogenesis & high expression, promotes angiogenesis \\
\hline miRNAs ${ }^{21}$ & high/low expression, promotes /inhibits angiogenesis & high/low expression, promotes /inhibits angiogenesis \\
\hline $\mathrm{AGEs}^{22}$ & & high expression, promotes/inhibits angiogenesis \\
\hline $\mathrm{gFGF}^{23}$ & & high expression, inhibits angiogenesis \\
\hline $\mathrm{PEDF}^{24}$ & low expression, inhibits angiogenesis & high expression, inhibits angiogenesis \\
\hline SHP- $\left.\right|^{25}$ & high/low expression, inhibits angiogenesis & high expression, inhibits angiogenesis \\
\hline Endostatin & low expression, inhibits angiogenesis & high expression, inhibits angiogenesis \\
\hline Angiostatin & low expression, inhibits angiogenesis & high expression, inhibits angiogenesis \\
\hline Pericytes & sparse, promotes angiogenesis & lost, stabilize new blood vessels \\
\hline $\mathrm{EPCs}^{26}$ & & decline in activity/number, promotes angiogenesis \\
\hline TAMs $^{27}$ & activated, promotes angiogenesis & \\
\hline $\mathrm{CAFs}^{28}$ & activated, promotes angiogenesis & \\
\hline $\mathrm{MSCs}^{29}$ & activated, promotes angiogenesis & inhibited, promotes angiogenesis \\
\hline Overall effect & Angiogenesis is enhanced & Angiogenesis is reduced \\
\hline
\end{tabular}

Notes: ' vascular endothelial growth factor; ${ }^{2}$ hypoxia inducible factor-I; ${ }^{3}$ nitric oxide; ${ }^{4}$ stromal cell-derived factor-I; ${ }^{5}$ the receptor CXC chemokine receptor type $4 ;{ }^{6}$ cyclooxygenase-2; ${ }^{7}$ microsomal prostaglandin E synthase-I; ${ }^{8}$ prostaglandin E2; ${ }^{9}$ matrix metalloproteinases; ${ }^{10}$ reactive oxygen species; ${ }^{11}$ fibroblast growth factor; ${ }^{12}$ plasminogen activator inhibitor-I; ${ }^{13}$ tumor necrosis factor-alpha; ${ }^{14}$ transforming growth factor; ${ }^{15}$ platelet-derived growth factor; ${ }^{16}$ Wingless and Int-related protein; ${ }^{17}$ angiopoietins 2; ${ }^{18}$ Insulin-like growth factor I; ${ }^{19}$ intercellular adhesion molecule - I; ${ }^{20}$ neuropilin I; ${ }^{21}$ microRNA; ${ }^{22}$ advanced glycation end products; ${ }^{23}$ basic fibroblast growth factor; ${ }^{24}$ platelet derived growth factor; ${ }^{25}$ SRc homology-2-domain-containing phosphatase-I; ${ }^{26}$ Endothelial precursor cells; ${ }^{27}$ tumor-associated macrophages; ${ }^{28}$ cancer associated fibroblasts; ${ }^{29}$ Mesenchymal stem cells. 
various pro-angiogenesis factors. Also, a high concentration of insulin decreases the bioavailability of NO, and the generation of AGEs and gFGF play important roles in this process. The effects of hypoxia and hyperglycemia on each factor, as well as the interaction between each factor, are shown in Figure 1.

In addition, abnormalities of well-known angiogenesis inhibitors such as platelet derived growth factor (PEDF), SRc homology-2-domain-containing phosphatase-1 (SHP1), endostatin ${ }^{38,47,58,85}$ and angiostatin ${ }^{38,47,86,87,89}$ are also important in the occurrence and development of these two diseases. For example, PEDF: high levels of PEDF are closely associated with diabetic retinopathy and wound healing. ${ }^{13,78}$ Similarly, low levels of PEDF in tumors promote their development and are associated with poor prognosis. ${ }^{79}$ SHP-1 is similar to PEDF in that it is highly expressed in diabetes and inhibits angiogenesis through various mechanisms. ${ }^{21}$ Low expression in tumors promotes angiogenesis. ${ }^{80}$ Other factors not mentioned in the previous article also play an important role in tumor and diabetes angiogenesis through their own pathways, such as Semaphorins ${ }^{81,82}$, mesenchymal stem cells (MSCs), ${ }^{30,38,51,88}$ IGF1, Leptin, ${ }^{20}$ IGAM $^{31,38,52,73}$ COX2/mPGES-1/PGE2 ${ }^{22,42} \mathrm{Nrp}^{5,83,84}$ miRNAs, etc. However, as shown in Table 1, the overall result is still an increase in factors that promote angiogenesis and a decrease in inhibitors in tumors, leading to a great deal of angiogenesis in tumors, whereas the opposite is true in diabetes.

In addition to the related factors mentioned in this paper, it can be speculated that the reason why angiogenesis is inhibited in diabetes but promoted in tumors may also be related to the change of receptor configuration caused by long-term exposure to the relevant specific microenvironment, which leads to the failure of the corresponding information transmission after the binding of VEGF and other molecules to the receptor. Even the binding rate of these molecules to the receptor decreases, and thus the conduction efficiency decreases. On the other hand, it can also be speculated that whether the related genes controlling the expression of these factors are changed in the microenvironment related to diabetes and cancer patients, thus leading to the change in the amount of the expression types of related factors.

However, at present, VEGF, FGF, and other factors still have many deficiencies and defects in the treatment of angiogenesis, no matter in diabetes or tumor, so they cannot become effective clinical treatment targets, and there is no treatment strategy for anti-tumor development and anti- diabetic complications that has been recognized by all clinical aspects.
Therefore, in the microenvironment of diabetes and tumors, there is still a long way to go for tumor therapy to inhibit angiogenesis and diabetes therapy to promote angiogenesis.

\section{Author Contributions}

All authors made substantial contributions to conception and design, acquisition of data, or analysis and interpretation of data; took part in drafting the article or revising it critically for important intellectual content; agreed to submit to the current journal; gave final approval of the version to be published; and agree to be accountable for all aspects of the work.

\section{Funding}

This work was supported as follows: the National Natural Science Foundation of China (82070455, 81770450); the Related Foundation of Jiangsu Province (BK20201225, WSN-044, LGY2018092, QNRC2016836)

\section{Disclosure}

The authors declare no conflicts of interest for this work and that there is no conflict of interest regarding the publication of this article.

\section{References}

1. Hu L, Dai SC, Luan X, Chen J, Cannavicci A. Dysfunction and therapeutic potential of endothelial progenitor cells in diabetes mellitus. J Clin Med Res. 2018;10(10):752-757. doi:10.14740/jocmr3581w.

2. Kazerounian S, Lawler J. Integration of pro- and anti-angiogenic signals by endothelial cells. J Cell Commun Signal. 2018;12 (1):171-179. doi:10.1007/s12079-017-0433-3.

3. Song P, Hai Y, Wang X, et al. Realgar transforming solution suppresses angiogenesis and tumor growth by inhibiting VEGF receptor 2 signaling in vein endothelial cells. Arch Pharm Res. 2018;41 (4):467-480. doi:10.1007/s12272-018-1014-6.

4. Miyazaki T, Akasu R, Miyazaki A. Calpain proteolytic systems counteract endothelial cell adaptation to inflammatory environments. Inflamm Regen. 2020;40(1):5. doi:10.1186/s41232-020-00114-x.

5. Yetkin-Arik B, Kastelein AW, Klaassen I, et al. Angiogenesis in gynecological cancers and the options for anti-angiogenesis therapy. Biochim Biophys Acta Rev Cancer. 2021;1875(1):188446. doi:10.1016/j.bbcan.2020.188446.

6. Rubin JB, Lagas JS, Broestl L, et al. Sex differences in cancer mechanisms. Biol Sex Differ. 2020;11(1):17. doi:10.1186/s13293020-00291-x

7. Zhang Y, Wang S, Dudley AC. Models and molecular mechanisms of blood vessel co-option by cancer cells. Angiogenesis. 2020;23 (1):17-25. doi:10.1007/s10456-019-09684-y.

8. Latacz E, Caspani E, Barnhill R, et al. Pathological features of vessel co- option versus sprouting angiogenesis. Angiogenesis. 2020;23 (1):43-54. doi:10.1007/s10456-019-09690-0.

9. De Francesco EM, Sims AH, Maggiolini M, Sotgia F, Lisanti MP, Clarke RB. GPER mediates the angiocrine actions induced by IGF1 through the HIF-1 $\alpha$ /VEGF pathway in the breast tumor microenvironment. Breast Cancer Res. 2017;19(1):129. doi:10.1186/s13058-0170923-5. 
10. Lin L, Wang Q, Xu F, et al. BML-111, the lipoxin A4 agonist, modulates VEGF or $\mathrm{CoCl} 2$-induced migration, angiogenesis and permeability in tumor- derived endothelial cells. Immunol Lett. 2021;230:27-35. doi:10.1016/j.imlet.2020.12.007.

11. Chen DY, Sun NH, Chen X, et al. Endothelium-derived semaphorin $3 \mathrm{G}$ attenuates ischemic retinopathy by coordinating $\beta$-catenindependent vascular remodeling. J Clin Invest. 2021;131(4):e135296. doi:10.1172/JCI135296.

12. Wirostko B, Wong TY, Rafael S. Vascular endothelial growth factor and diabetic complications[J]. Prog Retin Eye Res. 2008;27 (6):608-621. doi:10.1016/j.preteyeres.2008.09.002

13. Matsuoka M, Ogata N, Minamino K, Higuchi A, Matsumura M. High levels of pigment epithelium-derived factor in the retina of a rat model of type 2 diabetes. Exp Eye Res. 2006;82(1):172-178. doi:10.1016/j.exer.2005.05.011.

14. Zhao Q, Sun D, Li Y, Qin J, Yan J. Integrated analyses of IncRNAs microarray profiles and mRNA-lncRNA coexpression in smooth muscle cells under hypoxic and normoxic conditions. Biosci Rep. 2019;39(4):BSR20181783. doi:10.1042/BSR20181783.

15. Liu M, Galli G, Wang Y, et al. Novel therapeutic targets for hypoxia-related cardiovascular diseases: the role of HIF-1. Front Physiol. 2020;11:774. doi:10.3389/fphys.2020.00774.

16. Wang P, Liu S, Wang Z, Zhao H, Zhang X. Altered levels of circulating natural antibodies against VEGFR1-derived peptide in atherosclerosis. J Int Med Res. 2020;48(8):300060520948750. doi: $10.1177 / 0300060520948750$.

17. Shi X, Wei YT, Li H, et al. Long non-coding RNA H19 in atherosclerosis: what role? Mol Med. 2020;26(1):72. doi:10.1186/s10020020-00196-w

18. Jing L, Li S, Li Q. Akt/hypoxia-inducible factor-1 $\alpha$ signaling deficiency compromises skin wound healing in a type 1 diabetes mouse model. Exp Ther Med. 2015;9(6):2141-2146. doi:10.3892/ etm.2015.2394.

19. Tawfik MK, Mohamed MI. Exenatide suppresses 1,2-dimethylhydrazine-induced colon cancer in diabetic mice: effect on tumor angiogenesis and cell proliferation. Biomed Pharmacother. 2016;82:106-116. doi:10.1016/j.biopha.2016.05.005.

20. Wu Y, Liu Y, Dong Y, Vadgama J. Diabetes-associated dysregulated cytokines and cancer. Integr Cancer Sci Ther. 2016;3(1):370-378. doi:10.15761/ICST.1000173.

21. Carranza K, Veron D, Cercado A, et al. Cellular and molecular aspects of diabetic nephropathy; the role of VEGF-A. Nefrologia. 2015;35(2):131-138. doi:10.1016/j.nefro.2015.05.013.

22. Finetti F, Travelli C, Ercoli J, Colombo G, Buoso E, Trabalzini L. Prostaglandin E2 and cancer: insight into tumor progression and immunity. Biology (Basel). 2020;9(12):434. doi:10.3390/ biology9120434.

23. López-Díez R, Shen X, Daffu G, et al. Ager deletion enhances ischemic muscle inflammation, angiogenesis, and blood flow recovery in diabetic mice. Arterioscler Thromb Vasc Biol. 2017;37 (8):1536-1547. doi:10.1161/ATVBAHA.117.309714.

24. Hanna RM, Lopez EA, Hasnain H, et al. Three patients with injection of intravitreal vascular endothelial growth factor inhibitors and subsequent exacerbation of chronic proteinuria and hypertension. Clin Kidney J. 2019;12(1):92-100. doi:10.1093/ckj/sfy060.

25. Song K, Wu H, Rahman HN, et al. Endothelial epsins as regulators and potential therapeutic targets of tumor angiogenesis. Cell Mol Life Sci. 2017;74(3):393-398. doi:10.1007/s00018-016-2347-2.

26. Amadori M, Barone D, Scarpi E, et al. Dynamic contrast-enhanced ultrasonography (D-CEUS) for the early prediction of bevacizumab efficacy in patients with metastatic colorectal cancer. Eur Radiol. 2018;28(7):2969-2978. doi:10.1007/s00330-017-5254-5.

27. Höglander EK, Nord S, Wedge DC, et al. Time series analysis of neoadjuvant chemotherapy and bevacizumab-treated breast carcinomas reveals a systemic shift in genomic aberrations. Genome Med. 2018;10(1):92. doi:10.1186/s13073-018-0601-y.
28. Stadlbauer A, Roessler K, Zimmermann M, et al. Predicting glioblastoma response to bevacizumab through MRI biomarkers of the tumor microenvironment. Mol Imaging Biol. 2019;21(4):747-757. doi:10.1007/s11307-018-1289-5.

29. Bloom MJ, Jarrett AM, Triplett TA, et al. Anti-HER2 induced myeloid cell alterations correspond with increasing vascular maturation in a murine model of HER2+ breast cancer. BMC Cancer. 2020;20 (1):359. doi:10.1186/s12885-020-06868-4.

30. Suenaga M, Mashima T, Kawata N, et al. Serum IL- 8 level as a candidate prognostic marker of response to anti-angiogenic therapy for metastatic colorectal cancer. Int $J$ Colorectal Dis. 2021;36 (1):131-139. doi:10.1007/s00384-020-03748-y.

31. Choi H, Moon A. Crosstalk between cancer cells and endothelial cells: implications for tumor progression and intervention. Arch Pharm Res. 2018;41(7):711-724. doi:10.1007/s12272-018-1051-1.

32. Kes MMG, Van den Bossche J, Griffioen AW, Huijbers EJM. Oncometabolites lactate and succinate drive pro-angiogenic macrophage response in tumors. Biochim Biophys Acta Rev Cancer. 2020;1874(2):188427. doi:10.1016/j.bbcan.2020.188427.

33. Alsharabasy AM, Glynn SA, Pandit A. The role of extracellular matrix in tumour angiogenesis: the throne has NOx servants. Biochem Soc Trans. 2020;48(6):2539-2555. doi:10.1042/BST20200208.

34. Subotički T, Mitrović Ajtić O, Beleslin-čokić BB, et al. Angiogenic factors are increased in circulating granulocytes and CD34+ cells of myeloproliferative neoplasms. Mol Carcinog. 2017;56(2):567-579. doi: $10.1002 / \mathrm{mc} .22517$.

35. Janota J, Pomyje J, Toth D, et al. Expression of angiopoietic factors in normal and type-I diabetes human placenta: a pilot study. Eur J Obstet Gynecol Reprod Biol. 2003;111(2):153-156. doi:10.1016/ s0301-92115(03)00204-5

36. Safaeian L, Vaseghi G, Mirian M, Firoozabadi MD. The effect of pramlintide, an antidiabetic amylin analogue, on angiogenesis-related markers in vitro. Res Pharm Sci. 2020;15(4):323-330. doi:10.4103/ 1735-5362.293510.

37. Eelen G, de Zeeuw P, Simons M, Carmeliet P. Endothelial cell metabolism in normal and diseased vasculature. Circ Res. 2015;116 (7):1231-1244. doi:10.1161/CIRCRESAHA.116.302855.

38. Ahmadi M, Rezaie J. Tumor cells derived-exosomes as angiogenenic agents: possible therapeutic implications. J Transl Med. 2020;18 (1):249. doi:10.1186/s12967-020-5

39. Tuerxun H, Cui J. The dual effect of morphine on tumor development. Clin Transl Oncol. 2019;21(6):695-701. doi:10.1007/ s12094-018-1974-5.

40. Yu JW, Deng YP, Han X, Ren GF, Cai J, Jiang GJ. Metformin improves the angiogenic functions of endothelial progenitor cells via activating AMPK/eNOS pathway in diabetic mice. Cardiovasc Diabetol. 2016;15(1):88. doi:10.1186/s12933-016-0408-3.

41. Berezin AE. Endothelial progenitor cells dysfunction and impaired tissue reparation: the missed link in diabetes mellitus development. Diabetes Metab Syndr. 2017;11(3):215-220. doi:10.1016/j.dsx.2016.08.007.

42. Oba K, Hosono K, Amano H, et al. Downregulation of the proangiogenic prostaglandin E receptor EP3 and reduced angiogenesis in a mouse model of diabetes mellitus. Biomed Pharmacother. 2014;68(8):1125-1133. doi:10.1016/j.biopha.2014.10.022.

43. Zhou G, Han X, Wu Z, Shi Q, Bao X. Rosiglitazone accelerates wound healing by improving endothelial precursor cell function and angiogenesis in db/db mice. PeerJ. 2019;7:e7815. doi:10.7717/ peerj.7815.

44. Song Y, Xie X, Gao Y, Gu G, Wang P. Ultrasound-induced microbubble destruction promotes targeted delivery of adipose-derived stem cells to improve hind-limb ischemia of diabetic mice. $\mathrm{Am}$ J Transl Res. 2016;8(6):2585-2596.

45. Oak JH, Nakagawa K, Oikawa S, Miyazawa T. Amadori-glycated phosphatidylethanolamine induces angiogenic differentiations in cultured human umbilical vein endothelial cells. FEBS Lett. 2003;555 (2):419-423. doi:10.1016/s0014-5793(03)01237-7. 
46. Eiro N, González L, Martínez-Ordoñez A, et al. Cancer-associated fibroblasts affect breast cancer cell gene expression, invasion and angiogenesis. Cell Oncol. 2018;41(4):369-378. doi:10.1007/s13402018-0371-y.

47. Zhu M, Bi X, Jia Q, Shangguan S. The possible mechanism for impaired angiogenesis after transient focal ischemia in type 2 diabetic GK rats: different expressions of angiostatin and vascular endothelial growth factor. Biomed Pharmacother. 2010;64(3):208-213. doi:10.1016/j.biopha.2009.08.005.

48. Sethy C, Goutam K, Nayak D, et al. Clinical significance of a pvrl 4 encoded gene Nectin-4 in metastasis and angiogenesis for tumor relapse. $J$ Cancer Res Clin Oncol. 2020;146(1):245-259. doi:10.1007/s00432-019-03055-2.

49. Chen Y, Song Y, Du W, Gong L, Chang H, Zou Z. Tumor-associated macrophages: an accomplice in solid tumor progression. J Biomed Sci. 2019;26(1):78. doi:10.1186/s12929-019-0568-z.

50. Ferrara AL, Galdiero MR, Fiorelli A, et al. Macrophage-polarizing stimuli differentially modulate the inflammatory profile induced by the secreted phospholipase A2 group IA in human lung macrophages. Cytokine. 2021;138:155378. doi:10.1016/j.cyto.2020.155378.

51. Singh P, Sood NA. Combination of antigen retrieval methods significantly enhances the unmasking of tedious antigens of lymphangiogenesis and angiogenesis in canine mammary tumour. $J$ Biosci Med. 2017;5(12):44-55. doi:10.4236/jbm.2017.512006.

52. Sundararajan V, Sarkar FH, Ramasamy TS. Correction to: the versatile role of exosomes in cancer progression: diagnostic and therapeutic implications. Cell Oncol. 2018;41(4):463. doi:10.1007/s13402018-0396-2.

53. Zeferino RC, Mota NSRS, Grinevicius VMAS, et al. Targeting ROS overgeneration by N-benzyl-2-nitro-1-imidazole- acetamide as a potential therapeutic reposition approach for cancer therapy. Invest New Drugs. 2020;38(3):785-799. doi:10.1007/s10637-01900820-5.

54. Cao J, Liu X, Yang Y, et al. Decylubiquinone suppresses breast cancer growth and metastasis by inhibiting angiogenesis via the ROS/p53/ BAI1 signaling pathway. Angiogenesis. 2020;23 (3):325-338. doi:10.1007/s10456-020-09707-z.

55. Nigro $\mathrm{C}$, Leone A, Longo $\mathrm{M}$, et al. Methylglyoxal accumulation de-regulates HoxA5 expression, thereby impairing angiogenesis in glyoxalase 1 knock-down mouse aortic endothelial cells. Biochim Biophys Acta Mol Basis Dis. 2019;1865(1):73-85. doi:10.1016/j. bbadis.2018.10.014.

56. Abdulkhaleq LA, Assi MA, Noor MHM, Abdullah R, Saad MZ, Taufiq-Yap YH. Therapeutic uses of epicatechin in diabetes and cancer. Vet World. 2017;10(8):869-872. doi:10.14202/vetworld.2017.869-872.

57. Lekovic D, Gotic M, Skoda R, et al. Bone marrow microvessel density and plasma angiogenic factors in myeloproliferative neoplasms: clinicopathological and 42 molecular correlations. Ann Hematol. 2017;96(3):393-404. doi:10.1007/s00277-016-43-2890-9

58. Ghadir M, Khamseh ME, Panahi-Shamsabad M, et al. Cell proliferation, apoptosis, and angiogenesis in non- functional pituitary adenoma: association with tumor invasiveness. Endocrine. 2020;69 (3):596-603. doi:10.1007/s12020-020-02366-6.

59. Huang Y, Marui A, Sakaguchi H, et al. Sustained release of prostaglandin E1 potentiates the impaired therapeutic angiogenesis by basic fibroblast growth factor in diabetic murine hindlimb ischemia. Circ J. 2008;72(10):1693-1699. doi:10.1253/circj.cj-07-0960.

60. Hameed S, Bhattarai P, Dai Z. Nanotherapeutic approaches targeting angiogenesis and immune dysfunction in tumor microenvironment. Sci China Life Sci. 2018;61(4):380-391. doi:10.1007/s11427-0179256-1.

61. Huang XY, Huang ZL, Huang J, et al. Exosomal circRNA-100338 promotes hepatocellular carcinoma metastasis via enhancing invasiveness and angiogenesis. J Exp Clin Cancer Res. 2020;39(1):20. doi: $10.1186 /$ s 13046-020-9
62. Zhang Q, Wang W, Zhou Q, et al. Roles of circRNAs in the tumour microenvironment. Mol Cancer. 2020;19(1):14. doi:10.1186/s12943019-1125-9.

63. Ludwig N, Rubenich DS, $€$ Z, et al. Potential roles of tumor cell- and stroma cell-derived small extracellular vesicles in promoting a pro-angiogenic tumor microenvironment. Cancers (Basel). 2020;12 (12):3599. doi:10.3390/cancers 12123599

64. Maiese K. Moving to the rhythm with clock (circadian) genes, autophagy, mTOR, and SIRT1 in degenerative disease and cancer. Curr Neurovasc Res. 2017;14(3):299-304. doi:10.2174/ 1567202614666170718092010.

65. Nguyen VHL, Hough R, Bernaudo S, Peng C. Wnt/ß-catenin signalling in ovarian cancer: insights into its hyperactivation and function in tumorigenesis. J Ovarian Res. 2019;12(1):122. doi:10.1186/ s13048-019-0596-z.

66. Yang D, Li Q, Shang R, et al. WNT4 secreted by tumor tissues promotes tumor progression in colorectal cancer by activation of the Wnt/ $\beta$-catenin signalling pathway. J Exp Clin Cancer Res. 2020;39(1):251. doi:10.1186/s13046-020-01774-w.

67. Unterleuthner D, Neuhold P, Schwarz K, et al. Cancer-associated fibroblast-derived WNT2 increases tumor angiogenesis in colon cancer. Angiogenesis. 2020;23(2):159-177. doi:10.1007/s10456-01909688-8.

68. Solimando AG, Summa S, Vacca A, Ribatti D. Cancer-associated angiogenesis: the endothelial cell as a checkpoint for immunological patrolling. Cancers (Basel). 2020;12(11):3380. doi:10.3390/ cancers12113380.

69. Mouquet F, Cuilleret F, Susen S, et al. Metabolic syndrome and collateral vessel formation in patients with documented occluded coronary arteries: association with hyperglycaemia, insulin-resistance, adiponectin and plasminogen activator inhibitor-1. Eur Heart J. 2009;30(7):840-849. doi:10.1093/eurheartj/ehn569

70. Yoon CH, Choi YE, Koh SJ, Choi JI, Park YB, Kim HS. High glucose-induced jagged 1 in endothelial cells disturbs notch signaling for angiogenesis: a novel mechanism of diabetic vasculopathy. $\mathrm{J} \mathrm{Mol}$ Cell Cardiol. 2014;69:52-66. doi:10.1016/j.yjmcc.2013.12.006. PMID: 24361239.

71. Rodriguez YI, Campos LE, Castro MG, et al. Tumor necrosis factor receptor-1 (p55) deficiency attenuates tumor growth and intratumoral angiogenesis and stimulates CD8+ $\mathrm{T}$ cell function in melanoma. Cells. 2020;9(11):2469. doi:10.3390/cells9112469.

72. González Á, García de Durango C, Alonso V, et al. Distinct osteomimetic response of androgen-dependent and independent human prostate cancer cells to mechanical action of fluid flow: prometastatic implications. Prostate. 2017;77(3):321-333. doi:10.1002/pros.23270.

73. Nogués A, Gallardo-Vara E, Zafra MP, et al. Endoglin (CD105) and VEGF as potential angiogenic and dissemination markers for colorectal cancer. World J Surg Oncol. 2020;18(1):99. doi:10.1186/ s12957-020-01871-2.

74. Tanaka R, Masuda H, Fujimura S, et al. Quality-quantity control culture enhances vasculogenesis and wound healing efficacy of human diabetic peripheral blood CD34+ cells. Stem Cells Transl Med. 2018;7(5):428-438. doi:10.1002/sctm.17-0043

75. Fard D, Tamagnone L. Semaphorins in health and disease. Cytokine Growth Factor Rev. 2021;57:55-63. doi:10.1016/j. cytogfr.2020.05.006.

76. Tan S, Xia L, Yi P, et al. Exosomal miRNAs in tumor microenvironment. $J$ Exp Clin Cancer Res. 2020;39(1):67. doi:10.1186/s13046-020-01570-6.

77. Xie C, Li SY, Fang JH, Zhu Y, Yang JE. Functional long non-coding RNAs in hepatocellular carcinoma. Cancer Lett. 2021;500:281-291. doi:10.1016/j.canlet.2020.10.042.

78. Cheng R, Ma JX. Angiogenesis in diabetes and obesity. Rev Endocr Metab Disord. 2015;16(1):67-75. doi:10.1007/s11154-015-9310-7. 
79. Ansari D, Althini C, Ohlsson H, Bauden M, Andersson R. The Role of PEDF in pancreatic cancer. Anticancer Res. 2019;39 (7):3311-3315. doi:10.21873/anticanres.13473.

80. Wu C, Sun M, Liu L, Zhou GW. The function of the protein tyrosine phosphatase SHP-1 in cancer. Gene. 2003;306:1-12. doi:10.1016/ s0378-1119(03)00400-1. PMID: 12657462.

81. Hu S, Zhu L. Semaphorins and their receptors: from axonal guidance to atherosclerosis. Front Physiol. 2018;9:1236. doi:10.3389/ fphys.2018.01236.

82. Iyer AS, Chapoval SP. Neuroimmune semaphorin 4A in cancer angiogenesis and inflammation: a promoter or a suppressor? Int J Mol Sci. 2018;20(1):124. doi:10.3390/ijms20010124.

83. Zhu Q, Li J, Wu Q, et al. Linc-OIP5 in the breast cancer cells regulates angiogenesis of human umbilical vein endothelial cells through YAP1/Notch/NRP1 signaling circuit at a tumor microenvironment. Biol Res. 2020;53(1):5. doi:10.1186/s40659-0200273-0. PMID: 32046779; PMCID: 44 PMC7014737.

84. Babu M, Durga Devi T, Mäkinen P, et al. Differential promoter methylation of macrophage genes is associated with impaired vascular growth in ischemic muscles of hyperlipidemic and type 2 diabetic mice: genome-wide promoter methylation study. Circ Res. 2015;117 (3):289-299. doi:10.1161/CIRCRESAHA.115.306424.
85. Atta HM, El-Rehani MA, Raheim SA, Galal AM. Lowering homocysteine decreases levels and expression of $\operatorname{VEGF}(165)$ and endostatin. J Surg Res. 2008;146(2):202-210. doi:10.1016/j. jss.2007.04.038

86. Li T, Kang G, Wang T, Huang H. Tumor angiogenesis and anti-angiogenic gene therapy for cancer. Oncol Lett. 2018;16 (1):687-702. doi:10.3892/ol.2018.8733.

87. Ertürk İ, Sertoğlu E, Yeşildal F, Acar R, Özgürtaş T, Sağlam K. Angiostatin levels in diabetes mellitus patients receiving insulin treatment: associations with laboratory findings, comorbidities, and medications. Turk J Med Sci. 2018;48(6):1192-1199. doi:10.3906/ sag-1802-130.

88. Sakata N, Goto M, Yoshimatsu G, Egawa S, Unno M. Utility of co-transplanting mesenchymal stem cells in islet transplantation. World J Gastroenterol. 2011;17(47):5150-5155. doi:10.3748/wjg. v17.i47.5150.

89. Bai YY, Gao X, Wang YC, et al. Image-guided pro- angiogenic therapy in diabetic stroke mouse models using a multi-modal nanoprobe. Theranostics. 2014;4(8):787-797. doi:10.7150/thno.9525

\section{Publish your work in this journal}

Diabetes, Metabolic Syndrome and Obesity: Targets and Therapy is an international, peer-reviewed open-access journal committed to the rapid publication of the latest laboratory and clinical findings in the fields of diabetes, metabolic syndrome and obesity research. Original research, review, case reports, hypothesis formation, expert opinion and commentaries are all considered for publication. The manuscript management system is completely online and includes a very quick and fair peer-review system, which is all easy to use. Visit http://www.dovepress.com/testimonials.php to read real quotes from published authors.

Submit your manuscript here: https://www.dovepress.com/diabetes-metabolic-syndrome-and-obesity-targets-and-therapy-journal 